\title{
SIMULAÇÃO DO CRAQUEAMENTO TÉRMICO DE ÓLEO DE CANOLA, EMPREGANDO O SIMULADOR ASPEN HYSYS ${ }^{\circledast}$.
}

\author{
A. K. TAKAKURA ${ }^{1}$, E. C. $\operatorname{COSTA}^{1}$, N. T. MACHADO ${ }^{1}$, M. E. ARAÚHO 1, L.E.P. BORGES ${ }^{2}$. \\ ${ }^{1}$ Universidade Federal do Pará, Programa de Pós-Graduação em Engenharia de Recursos Naturais da \\ Amazônia \\ ${ }^{2}$ Instituto Militar de Engenharia, Departamento de Engenharia Quimica.
}

E-mail para contato: alberto_koji@hotmail.com

\begin{abstract}
RESUMO - Este trabalho tem como objetivo simular o craqueamento térmico de óleo de canola. O simulador utilizado foi o Aspen HYSYS. O mecanismo de reação de craqueamento adotado, de acordo com a literatura, consiste em cinco etapas distintas: decomposição dos triglicerídeos, decomposição dos ácidos graxos, decomposição do cetano e da acroleína, formação do coque e formação do produto final. As condições operacionais utilizadas foram: temperatura de $400{ }^{\circ} \mathrm{C}$ e pressão $1 \mathrm{~atm}$, escolhidas com base em dados de literatura. Utilizou-se óleo de canola como matéria-prima para as reações, cujos produtos são OLP (Produto orgânico líquido), gás e óleo residual. Os resultados da simulação foram analisados e comparados com os dados disponíveis na literatura.
\end{abstract}

\section{INTRODUÇÃO}

O interesse crescente por novas fontes alternativas de energia no Brasil e no mundo, que sejam menos poluentes que os combustíveis de origem fóssil, têm levado à pesquisas com foco na substituição gradual destes combustíveis. Além da preocupação com o meio ambiente no que tange à proteção e conservação ambiental, existe ainda a perspectiva de esgotamento das reservas de origem fóssil nas próximas décadas. Neste contexto, vários pesquisadores vêm se concentrando em fontes renováveis de combustíveis líquidos para substituir ou amenizar o consumo de produtos fósseis no futuro, com destaque para derivados da biomassa, em razão da sua natureza renovável, ampla disponibilidade, biodegradabilidade e baixo custo (Lhamas, 2013).

Dentre as diversas rotas tecnológicas investigadas, se destaca o craqueamento, um método eficiente para a produção de biocombustíveis. A reação de craqueamento, ou pirólise, de triglicerídeos consiste no processo de quebra das cadeias carbônicas dos triésteres de origem animal ou vegetal pelo aumento da temperatura do sistema, resultando em uma mistura de compostos constituída, em sua maioria, por hidrocarbonetos lineares de diferentes pesos moleculares (Ma e Hanna, 1999). Os produtos do processo de craqueamento incluem parafinas e olefinas cíclicas e lineares, além de produtos oxigenados, tais como ácidos carboxílicos, cetonas, aldeídos, ésteres e acroleína (Maher e Bessler, 2007). Entretanto o tamanho das moléculas formadas depende do tipo de triglicerídeo 


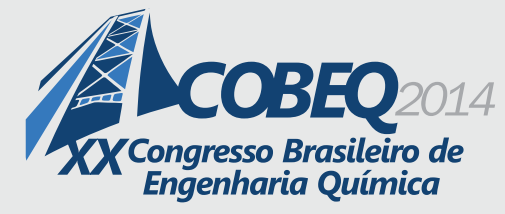

utilizado e das reações consecutivas ao craqueamento, sendo, dessa forma, a reação de pirólise de difícil caracterização. Nesse sentido, há uma alta complexidade no controle da formação dos produtos que depende de diversos fatores como temperatura, atmosfera, presença de catalisador, etc. (Barreto, 2009).

Chang e Wan (1947), realizaram um estudo detalhado do craqueamento de óleo e propuseram um mecanismo de reação composto por duas etapas distintas. $\mathrm{Na}$ primeira etapa, são formados principalmente ácidos carboxílicos decorrentes do rompimento da ligação C-O da parte glicerídica e o resto da cadeia do óleo ou gordura, esta etapa é conhecida como craqueamento primário. A segunda etapa, caracterizada pela degradação dos ácidos produzidos durante a primeira etapa, recebe o nome de craqueamento secundário (Lhamas, 2013).

Este trabalho teve como objetivo simular o craqueamento térmico do óleo de Canola utilizando o simulador comercial Aspen Hysys. Para isto, foi adotado o mecanismo de reação proposto por Chang e Wan (1947), e os resultados comparados aos obtidos, experimentalmente, por Idem et al. (1996) que realizou o craqueamento de óleo de Canola.

\section{Modelo Termodinâmico}

O sucesso da modelagem de processos depende da precisão da descrição das propriedades termodinâmicas e das condições de equilíbrio de fases. $\mathrm{O}$ cálculo do equilíbrio de fases pode ser baseado na aplicação de equações de estado (EDE). Assim, uma única equação é usada para representar todas as fases fluidas (Teribeli, et al. 2006). As equações de estado cúbicas de SoaveRedlich-Kwong (SRK) e Peng-Robinson (PR) e suas modificações são as mais amplamente utilizadas na indústria, no entanto, existem outras equações que podem ser utilizadas para descrever o equilíbrio de fases de uma mistura e, portanto, para descrever processos. Entre elas citamos a equação de TwuSim-Tassone (TST). A equação TST é uma EDE cúbica de dois parâmetros, proposta visando uma melhor predição de densidades líquidas de hidrocarbonetos pesados e componentes polares. A função alfa generalizada para TST é uma função linear do fator acêntrico a uma temperatura reduzida constante, e não uma função de quarta ordem ou sexta ordem, tal como sugerido por Soave e outros pesquisadores. A vantagem de uma função linear do fator acêntrico fica evidente na extrapolação da função alfa para hidrocarbonetos pesados, frações de petróleo, e gases condensados. A função alfa generalizada, quando usada com TST permite uma previsão precisa da pressão do vapor a partir do ponto triplo para o ponto crítico de hidrocarbonetos (Twu et al, 2002).

A EDE cúbica TST é representada pela equação 1.

$$
P=\frac{R T}{v-b}-\frac{a}{(v+3 b)(v-0,5 b)}
$$

Os valores de $\mathrm{a}$ e b à temperatura crítica são encontrados pela primeira e segunda derivadas da pressão em relação ao volume, resultando em: 


$$
a_{c}=\frac{0,470507 R^{2} T_{c}^{2}}{P_{c}} \quad, \quad b_{c}=\frac{0,0740740 R T_{c}}{P_{c}} \quad \text { e } \quad Z_{c}=0,296292
$$

Onde: o subscrito $c$ refere-se ao ponto crítico. Vale salientar que o valor de $Z c$ com SRK e PR tem dimensão maior que 0,3 e para TST é ligeiramente abaixo desse valor.

\subsection{Simulação do Processo de Craqueamento}

No presente trabalho, é realizada a simulação do processo de craqueamento térmico de óleo de canola utilizando o simulador ASPEN HYSYS ${ }^{\circledR}$. O projeto foi concebido segundo algumas premissas e considerações: o modelo termodinâmico selecionado para realizar as simulações foi o TST (Twu-Sim-Tassone); a capacidade da planta é de $1000 \mathrm{~kg} / \mathrm{h}$; temperatura de $400^{\circ} \mathrm{C}$ e pressão igual a $101,3 \mathrm{kPa}$; os reatores utilizados foram reatores de equilíbrio com taxa de conversão de 98\%; para a corrente de alimentação foi admitida como único componente a Trioleína; como o craqueamento é caracterizado por uma serie de reações para a realização da simulação optou-se pela utilização vários reatores, um para cada reação envolvida.

As propriedades críticas e fator acêntrico dos componentes da mistura, cadastrados como hipotéticos no simulador, foram estimados pelo método de contribuição de grupos proposto por Constantinou e Gani (1994) e Constantinou et al. (1995).

Como descrito anteriormente, neste trabalho utilizou o mecanismo proposto por Chang e Wan (1947). O mecanismo consiste, resumidamente, nas cinco etapas descritas a seguir: decomposição dos triglicerídeos, decomposição dos ácidos graxos, decomposição do cetano e da acroleína, a formação do coke e a formação do produto final. As reações do craqueamento são sequenciais, ou seja, uma reação depende das demais. No entanto, na formação do produto final as reações são independentes e reversíveis. Na decomposição da molécula de triglicerídeos são formadas duas moléculas de ácidos graxos e uma molécula de cetano. No processo seguinte, há a decomposição de ácidos graxos onde ocorrem duas reações, uma formando moléculas de $\mathrm{CO}_{2} \mathrm{e}$ hidrocarboneto e, outra onde ocorre a formação de moléculas de $\mathrm{CO}_{2}$, água e cetona. $\mathrm{Na}$ decomposição do cetano e da acroleína, os produtos das reações são hidrocaboneto, monóxido de carbono e a acroleína. As cetonas e os hidrocarbonetos resultantes da decomposição de ácidos graxos e do cetano serão utilizados na formação dos produtos finais (bio-óleo) e a acroleína é um dos produtos finais do craqueamento. A formação do coke ocorre independentemente das outras reações, ou seja, ele pode ser formado como produto de qualquer uma das três etapas descritas anteriormente. O coke também é um dos produtos finais do craqueamento. A formação do produto final consiste nas seguintes reações: decomposição das parafinas, aromatização ou ciclização das parafinas, depolimerização das olefinas, ciclização das olefinas e alquilização. 
A Figura 1 apresenta o fluxograma da planta de craqueamento térmico inicial de óleo de canola.

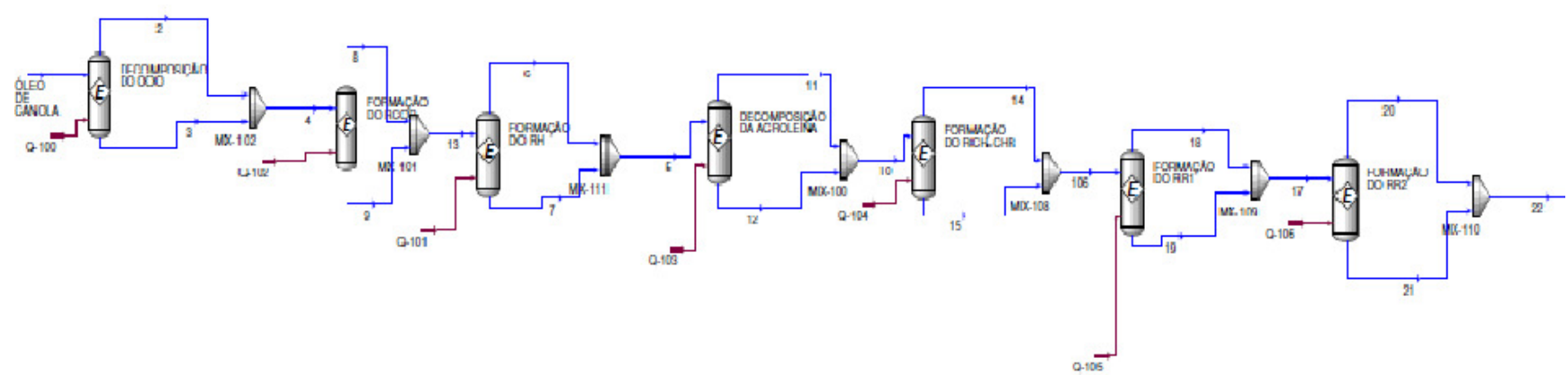

Figura 1- Planta do processo de craqueamento térmico inicial de óleo de canola.

\section{RESULTADOS E DISCUSSÕES}

Nas tabelas 1 está apresentada as frações mássicas dos produtos de Idem et al. (1996), e da simulação realizada com o ASPEN HYSYS. Pode ser verificado que a simulação obteve maior fração mássica de gases, o que pode está relacionado ao modelo termodinâmico utilizado na simulação (TST), visto que este modelo é especialmente recomendado para hidrocarbonetos de cadeias longas. Outro fator que pode explicar as diferenças é o fato da matéria-prima utilizada na simulação, o óleo de canola, ter sido representado apenas pela Trioleína.

Tabela1 - Porcentagem mássica dos produtos obtidos no craqueamento térmico do óleo de canola

\begin{tabular}{ccc}
\hline \multirow{2}{*}{ Produto } & Idem et al. (1996) & ASPEN HYSYS \\
\cline { 2 - 3 } & & $(\%)$ \\
Gás & 55,8 & 76,14 \\
PLO & 34,4 & 13,09 \\
Coke & 3,9 & - \\
Óleo Residual & 1,6 & 7,02 \\
Fração Não-reconhecida & 4,3 & 3,75 \\
\hline Total & 100 & 100 \\
\hline Conversão de Óleo de Canola & 98,4 & 98,0 \\
\hline
\end{tabular}

$\mathrm{Na}$ Tabela 2 estão apresenta as temperaturas, frações de vapor e líquido e as porcentagens em massa do PLO, nas correntes finais da decomposição do óleo e do produto final, da planta de simulação desenvolvida. 
Na corrente final da decomposição final do óleo, foi verificada a diferença entre a fração de vapor e a fração do líquido. Este fato ocorreu devido a alta temperatura $\left(400^{\circ} \mathrm{C}\right)$ do processo de craqueamento, no qual ultrapassa a temperatura de ebulição da maioria das substâncias. Foi verificado também, a fração mássica da trioleína $(\mathrm{OOO})$, ácido oleico e acroleína que foram decompostas em toda sua totalidade. A substância R-CO-R teve número bem expressivo, com isso foi adotado outro mecanismo de reação para a formação de etanol e n-pentano.

$\mathrm{Na}$ corrente do produto final, teve a sua temperatura reduzida para $25^{\circ} \mathrm{C}$ para ser melhor analisada. Foi verificada com a redução de temperatura ocasionou duas fases distintas, uma de vapor e outra de líquido, sendo que na fase líiquida, o simulador considerou também uma fase aquosa.

Foi verificada também, a formação do produto, onde as substâncias formadas não ultrapassaram $8 \%$ da massa total do produto de cada produto.

Tabela 1 - Propriedades e Composição das Correntes Principais.

\begin{tabular}{|c|c|c|}
\hline Correntes & Decomposição Final do Óleo & Produto Final \\
\hline Fração de vapor & 0,8177 & 0,7614 \\
\hline \multirow{2}{*}{ Fração de líquido } & \multirow{2}{*}{0,1823} & 0,2011 \\
\hline & & $0,0375^{* *}$ \\
\hline Temperatura $\left({ }^{\circ} \mathrm{C}\right)$ & 351,16 & 25 \\
\hline Vazão mássica (kg/h) & 1000 & 1000 \\
\hline \multicolumn{3}{|c|}{ Composição Mássica } \\
\hline OOO* & 0,0000 & 0,0000 \\
\hline OleicAcid & 0,0003 & 0,0003 \\
\hline Acrolein & 0,0000 & 0,0000 \\
\hline Cetanos* & 0,0060 & 0,0060 \\
\hline $\mathrm{CO}$ & 0,0673 & 0,0673 \\
\hline Ethane & 0,0410 & 0,0438 \\
\hline $\mathrm{CO}_{2}$ & 0,0507 & 0,0507 \\
\hline $\mathrm{H}_{2} \mathrm{O}$ & 0,0199 & 0,0199 \\
\hline $\mathrm{R}-\mathrm{CO}-\mathrm{R}^{*(\mathrm{a})}$ & 0,3859 & 0,0077 \\
\hline $\mathrm{RH}^{*}(\mathrm{a})$ & 0,0110 & 0,0110 \\
\hline $\mathrm{R}-\mathrm{CH}=\mathrm{CH}-\mathrm{R}^{*}$ & 0,2617 & 0,0052 \\
\hline $\mathrm{R}-\mathrm{R} *(\mathrm{a})$ & 0,1561 & 0,0102 \\
\hline $\mathrm{CH}_{2} \mathrm{CO}^{*}$ & 0,0002 & 0,0002 \\
\hline 1-Butene & 0,0000 & 0,0000 \\
\hline Methane & 0,0000 & 0,0016 \\
\hline 2-Metil-1-Buteno* & 0,0000 & 0,0384 \\
\hline n-Hexane & 0,0000 & 0,0001 \\
\hline Benzene & 0,0000 & 0,0044 \\
\hline
\end{tabular}


Tabela 2 - Propriedades e Coposição das Correntes Principais (cont.)

\begin{tabular}{|c|c|c|}
\hline Hydrogen & 0,0000 & 0,0040 \\
\hline n-Butane & 0,0000 & 0,0336 \\
\hline 1,3-Butadienos* & 0,0000 & 0,0000 \\
\hline $\mathrm{C}_{23} \mathrm{H}_{46} *$ & 0,0000 & 0,0006 \\
\hline Propene & 0,0000 & 0,0202 \\
\hline $\mathrm{C}_{33} \mathrm{H}_{68} *$ & 0,0000 & 0,0006 \\
\hline $\mathrm{n}-\mathrm{C} 16$ & 0,0000 & 0,0003 \\
\hline $\mathrm{C}_{17} \mathrm{H}_{34} *$ & 0,0000 & 0,0003 \\
\hline n-Decane & 0,0000 & 0,0122 \\
\hline n-Nonane & 0,0000 & 0,0002 \\
\hline $\mathrm{C}_{24} \mathrm{H}_{48} *$ & 0,0000 & 0,0292 \\
\hline $\mathrm{C}_{27} \mathrm{H}_{54} *$ & 0,0000 & 0,0007 \\
\hline n-Pentane & 0,0000 & 0,2309 \\
\hline $\mathrm{C}_{28} \mathrm{H}_{56} *$ & 0,0000 & 0,0000 \\
\hline $\mathrm{C}_{29} \mathrm{H}_{58} *$ & 0,0000 & 0,0376 \\
\hline Propane & 0,0000 & 0,0041 \\
\hline $\mathrm{C}_{30} \mathrm{H}_{60} *$ & 0,0000 & 0,0008 \\
\hline $\mathrm{C}_{31} \mathrm{H}_{62} *$ & 0,0000 & 0,0008 \\
\hline Cyclohexane & 0,0000 & 0,0028 \\
\hline Cyclopentane & 0,0000 & 0,0769 \\
\hline Cyclopropane & 0,0000 & 0,0002 \\
\hline Carbon & 0,0000 & 0,0000 \\
\hline 1,2-Di-Metil-ciclopropano* & 0,0000 & 0,0005 \\
\hline Fluoranthene & 0,0000 & 0,0122 \\
\hline 1-Hepteno* & 0,0000 & 0,0244 \\
\hline 1 1,5Dimetil-Hexil -4- 4Metil-Pentil Ciclohexano* & 0,0000 & 0,0003 \\
\hline Indene & 0,0000 & 0,0100 \\
\hline isobutano* & 0,0000 & 0,0000 \\
\hline isobutileno* & 0,0000 & 0,0000 \\
\hline n-Octane & 0,0000 & 0,0304 \\
\hline 2,4-Octadienos* & 0,0000 & 0,0000 \\
\hline Naftaleno* & 0,0000 & 0,0000 \\
\hline 1,3-Pentadienos* & 0,0000 & 0,0005 \\
\hline 1-Octeno* & 0,0000 & 0,0096 \\
\hline 1-Pentil-2-Propril-Ciclopropano* & 0,0000 & 0,0000 \\
\hline i-Butane & 0,0000 & 0,0263 \\
\hline n-Heptane & 0,0000 & 0,0000 \\
\hline 1-Ciclo-pentano* & 0,0000 & 0,0000 \\
\hline $\mathrm{C}_{32} \mathrm{H}_{64} *$ & 0,0000 & 0,0462 \\
\hline 1-Dodeceno* & 0,0000 & 0,0150 \\
\hline Ciclopentane* & 0,0000 & 0,0000 \\
\hline
\end{tabular}




\begin{tabular}{ccc}
\hline 1-Penteno* & 0,0000 & 0,0240 \\
2,4-hexadienos* & 0,0000 & 0,0435 \\
Ethanol & 0,0000 & 0,0346 \\
\hline *Componentes cadastrados como Hipotéticos. **Fração da Fase A Auosa na corrente ${ }^{(a)}$ R representa radical $C_{17} H_{34}$
\end{tabular}

\section{CONCLUSÃO}

Este trabalho teve como objetivo simular o craqueamento térmico de óleo de canola, utilizando o simulador Aspen HYSYS. Foram avaliadas as diferenças entre os produtos do craqueamento obtidos por de Idem et al. (1996) e os da simulação.

Foi observada uma diferença significativa nos resultados da simulação, o que pode ser em decorrência da utilização do pacote termodinâmico e da caracterização de algumas substâncias que influenciaram os resultados.

\section{REFERÊNCIAS}

BARRETO, C. C. K. Avaliação da estabilidade de bio-óleo obtido a partir de óleo de soja durante armazenamento. 2009. 100 f. Dissertação (Mestrado em Química)- Universidade de Brasília. Brasília,DF,2009.

CHANG, C.C., WAN, S.W., 1947. China's Motor Fuels from Tung Oil. Ind. Eng. Chem. 39, 15431548.

CONSTANTINOU, L.; GANI, R. New group contribution method for estimating properties of pure compounds. American Institute of Chemical Engineers Journal. v. 40, p. 1697-1710, 1994.

CONSTANTINOU, L.; GANI, R.; O'CONNELL, J. P. Estimation of the acentric factor and the liquid molar volume at $298 \mathrm{~K}$ using a new group contribution method. Fluid Phase Equilibria. v. 103, p. 11-22, 1995.

IDEM, R. O., KATIKANENI, S. P. R., BAKHSHI, N. N., 1996. Thermal cracking of canola oil: reaction products in the presence and absence of steam. Energy Fuels 10 (6), 1150-1162.

LHAMAS, D. E. L. Estudo do Processo de Craqueamento Termocatalítico do Óleo de Palma (Elaeis guineensis) e do Óleo de Buriti (Mauritia flexuosa L.) para Produção de Biocombustível. 2013. 216 f. Tese (Doutorado em Engenharia de Recursos Naturais) - Universidade Federal do Pará. Belém - PA, 2013.

MA, F.; HANNA, M. A. Biodiesel production A review. Bioresource Tecnology, v. 70, p. 1-15, 1999. 
MAHER, K. D.; BRESSLER, D. C. Bioresource Technology, 2007, 98, 2351-2368.

TERIBELE, T.; SANTOS, J. L.; ARAÚJO, M. E. Simulação De Uma Unidade De Turbo-Expansão De Gás Natural Empregando Diferentes Equações De Estado. In: Cobeq 2006 - XVI Congresso Brasileiro de Engenharia Química e III Congresso Brasileiro de Termodinâmica Aplicada, 2006, Santos.

TWU, C. H., et. al., "A Versatile Liquid Activity Model for SRK, PR and A New Cubic Equation of State TST," Fluid Phase Equilibria, 194-197, pp. 385-399 (2002). 